\title{
ACUTE TOXICITY OF PIERISIN-1, A CYTOTOXIC PROTEIN FROM PIERIS RAPAE, IN MOUSE AND RAT
}

\author{
Atsushi SHIGA, Susumu KAKAMU, Yutaka SUGIYAMA, Maki SHIBATA, \\ Eriko MAKINO and Makoto ENOMOTO \\ Biosafety Research Center, Foods, Drugs and Pesticides (An-Pyo Center), \\ 582-2 Shioshinden, Iwata-shi, Shizuoka 437-1213, Japan
}

(Received December 26, 2005; Accepted January 23, 2006)

\begin{abstract}
To investigate the toxicity of pierisin-1, a cytotoxic protein present in the cabbage butterfly, Pieris rapae, pierisin-1 was administered via intraperitoneally in mice and rats and the effects examined. Common findings in these experiments were hypoactivity with a gradual decrease in body weight due to decreased food intake, relative polycythemia with low serum albumin concentration and atrophy of the thymus, spleen, seminal vesicles and adipose tissue. Characteristic findings were diarrhea, fusion and atrophy of the villi and dilatation of the crypts in the small intestine at $6-100 \mu \mathrm{g} / \mathrm{kg}$ in BALB/ c mice as well as elevation of LDH activity and creatinine value, hemolysis and renal and hepatic injuries at 1,000 and $10,000 \mu \mathrm{g} / \mathrm{kg}$ in BALB/c mice. In the case of ICR mice, severer renal injury was observed. On the other hand, in Fischer 344/Du rats, sudden stop of food intake, elevation of both AST and ALT activities, interlobar adhesion of the right hepatic lobe, capsular thickening, septal fibrosis and single cell necroses of subcapsular hepatocytes in the liver and basophilic tubules in the kidneys were observed. Oral administration of pierisin-1 at a dose of $10,000 \mu \mathrm{g} / \mathrm{kg}$ in BALB/c mice did not exert any obvious effects. Thus, existence of species and strain differences in toxicity of pierisin- 1 to animals was demonstrated.
\end{abstract}

KEY WORDS: Pierisin-1, Cytotoxic protein, Acute toxicity, Rat, Mouse

\section{INTRODUCTION}

Pierisin-1 is a $98-\mathrm{kDa}$ protein isolated from the cabbage butterfly, Pieris rapae, exhibits strong cytotoxicity to mammalian cells (Koyama et al., 1996; Watanabe et al., 1998). The $\mathrm{IC}_{50}$ values to a variety of cell lines range from $0.043 \mathrm{ng} / \mathrm{ml}$ to $270 \mathrm{ng} / \mathrm{ml}$ (Kono et al., 1999; Kanazawa et al., 2001). Pierisin-1 induces apoptosis to most cells, including HeLa cells, within 48 hr (Watanabe et al., 1998; Kono et al., 1999). Activation of caspases and release of cytochrome $\mathrm{c}$ have been documented (Kanazawa et al., 2002a) and overexpression of Bcl-2 protein in HeLa cells results in acquisition of resistance, so that, pierisin-1-induced apoptosis may be mediated primarily via a mitochondrial pathway involving Bcl-2 and caspases (Kanazawa et al., 2002a).

Amino acid sequence similarities suggested that pierisin-1 is an ADP-ribosylating toxin (Kanazawa et al., 2001). Such bacteria-derived ADP-ribosylating toxins feature an enzyme domain exhibiting mono (ADP-ribosyl) transferase activity and a receptor-binding domain allowing entry into target cells (Kanazawa et al., 2001; Watanabe et al., 1999). Although all of the acceptor molecules for ADP-ribosyl moieties catalyzed by bacteria-derived ADP-ribosylating toxins so far reported are proteins, pierisin-1 was found to transfer the moiety to the $\mathrm{N}-2$ position of guanine bases in DNA (Takamura-Enya et al., 2001). Thus, pierisin-1 is classified as a guanine-specific ADP-ribosylating toxin. Moreover, it is known to induce mutations in the HPRT gene in CHL cells (Totsuka et al., 2003). However, damage signaling and mechanisms of apoptosis induction caused by the modified DNA remain to be clarified.

Immunological and structural analyses of fractionated HeLa cellular components have demonstrated receptors for pierisin-1 to be neutral glycosphingolipids, globotriaosylceramide (Gb3) and globotetraocylceramide (Gb4) (Matsushima-Hibiya et al., 2003).

Correspondence: Atsushi SHIGA (E-mail: siga@anpyo.or.jp) 
Globopentaosylceramide (Gb5) also exhibits receptor activity for pierisin-1, and such glycolipids capable of binding to pierisin-1, including Gb3 and Gb4, are abundant in pierisin-1-sensitive cell lines, HeLa, TMK-1 and MCF-7, whereas they are lacking in the insensitive cell line, MEB4 (Matsushima-Hibiya et al., 2003). These observations indicate that the amount of receptor in cells is one of the main factors affecting sensitivity.

Despite the variation in strength, the cytotoxicity suggests that pierisin-1 could be a potent mammalian toxin with significant antitumor activity against certain cancers. Indeed, a single intraperitoneal injection of pierisin-1 suppressed HeLa tumor growth in BALB/c nude mice, and in a preliminary experiment the dose of $10 \mu \mathrm{g} / \mathrm{kg}$ i.p. was sufficient to kill the animals (Kanazawa et al., 2002b).

The present study, carried out to determine acute toxicity in detail, included pathological and biochemical analyses. As expected, pierisin-1 exhibited strong toxicity to BALB/c mice, ICR mice and Fischer 344 rats on intraperitoneal injection. Several strain/speciesspecific lesions such as dilatation of small intestinine at low dose $(6-100 \mu \mathrm{g} / \mathrm{kg})$ and hemolysis at high dose $(1,000$ and $10,000 \mu \mathrm{g} / \mathrm{kg})$ in BALB/c mice, renal injury in ICR mice at $30 \mu \mathrm{g} / \mathrm{kg}$ and emaciation due to no intake of food in Fischer 344 rats were observed. Possible target organs and cause of death are discussed.

\section{MATERIALS AND METHODS}

\section{Test substance}

Pierisin-1 was supplied from the National Cancer Center Research Institute (Dr. Kanazawa, Dr. Watanabe and Dr.Wakabayashi).

\section{Study outline and animals}

The present study encompassed five experiments. Protocols for each experiment, including the number of animals per group, animal age, and the doses and administration route used are presented in Table 1 . The study objects in experiments 1-4 were as follows. Experiment 1: single intraperitoneal administration with BALB/c mice (Japan Slc Inc., Shizuoka, Japan) at doses of 3, 6, 10, 30, 100, 1,000 and 10,000 $\mu \mathrm{g} / \mathrm{kg}$. Experiment 2: single oral administration with BALB/c mice at a dose of $10,000 \mu \mathrm{g} / \mathrm{kg}$. Experiment 3: single intraperitoneal administration with ICR mice (Japan Slc Inc.) at a dose of $30 \mu \mathrm{g} / \mathrm{kg}$. Experiment 4: singledose intraperitoneal administration with F344 rats (Japan Charles River Inc., Kanagawa, Japan) at doses of 10 and $30 \mu \mathrm{g} / \mathrm{kg}$. Pierisin-1, purified from the larvae of Pieris rapae as described previously (Watanabe $e t$ al., 1998) were dissolved in physiological saline with a volume of $0.1 \mathrm{~mL}$ per $20 \mathrm{~g}$ per body weight.

In Experiments 1, 3 and 4, the main general effects in mice and rats administered pierisin-1 was a decrease in body weight resulting from a decrease of food intake. Whether lesions observed in the pierisin1-treated animals were due to a decrease of food intake and what the cause of the disturbance of food intake needs to be investigated. Therefore, to clarify the direct effects of pierisin-1 on the animal body overall, fasting experiments with $\mathrm{BALB} / \mathrm{c}$ mice and $\mathrm{F} 344$ rats were carried out and designated as experiment 5.

All these experiments were carried out according to the Guidances for Animal Testing at An-Pyo Center.

\section{Housing conditions}

Animals were housed individually in aluminum cages with stainless steel wire mesh fronts and floors placed over an automatic water- flushing breeding rack in an air-conditioned animal room (temperature, $23 \pm 3^{\circ} \mathrm{C}$; relative humidity, $55 \pm 20 \%$; lighting cycle, 12 hr light-dark, illumination at intensity of 150-300 lx) with a barrier system. The animals were given commercial diet MF (Oriental Yeast Co., Ltd. Tokyo, Japan) and tap water ad libitum. Animal husbandry and handling were conducted in accordance with the AnPyo Center's animal use and care guidelines.

\section{Examinations}

Clinical observation was performed and body weight and food consumption were measured once a day, at the same time each day during the experimental period. Blood sampling for clinical laboratory tests was performed just before necropsy on all moribund and schedule-sacrificed animals, and thereafter pathological examinations were conducted. All major organs were removed and fixed in 10\% phosphate buffered formalin, processed routinely, and embedded in paraffin. Paraffin sections $3 \mu \mathrm{m}$ in thick were cut and stained with hematoxylin and and eosin. Parameters of clinical laboratory tests for each experiment are presented in Table 1.

\section{RESULTS}

The time-courses for death and mortality and the representative data in each experiment are summarized in Table 2. Changes in mean body weights and mean food consumption in Experiment 1 are presented in 
Acute toxicity of pierisin-1.

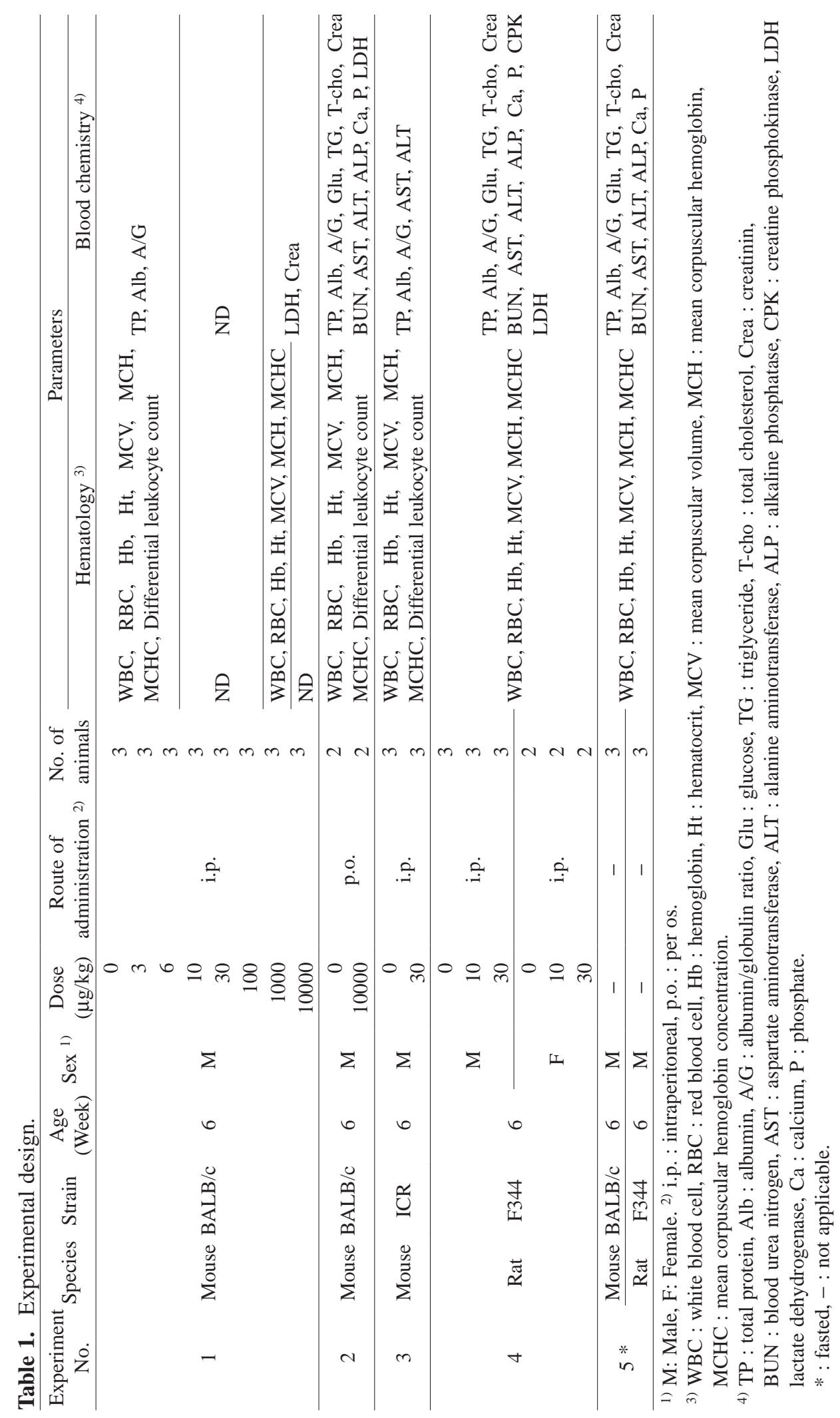


Figs. 1 and 2. Changes in mean body weights in Experiment 3 and 4 are presented in Figs. 3 and 4.

\section{Experiment 1}

Time-courses of change in mean body weight and food consumption are presented in Figs. 1 and 2, respectively. The interval from dosing until death was 7 days in the $6 \mu \mathrm{g} / \mathrm{kg}$ group and 1 day in the $10,000 \mu \mathrm{g} /$ $\mathrm{kg}$ group. $\mathrm{LD}_{100}$ in BALB/c mice was $30 \mu \mathrm{g} / \mathrm{kg}$. The dose and the time-course of death were well related (Table 2). Suppression of body weight gain due to decrease in food consumption occurred and was followed by the appearance of clinical signs. The interval from onset of clinical signs till death was approximately 1 day, irrespective of the dose. At doses of 6$100 \mu \mathrm{g} / \mathrm{kg}$, the first clinical sign was hyperactivity, followed by hypoactivity, and mice in the $10 \mu \mathrm{g} / \mathrm{kg}$ group showed diarrhea and abdominal distension. In addition, adoption of a hunchback position was also observed in mice in the $30 \mu \mathrm{g} / \mathrm{kg}$ group, suggesting the presence of abdominal pain. In clinical laboratory tests, high hematocrit value and red blood cell (RBC) counts were noted, whereas serum albumin concentrations were low. Therefore, these hematological findings were considered indicative of relative polycythemia with low albumin. This was a characteristic finding in pierisin-1-treated mice and rats as described here and after. At necropsy, atrophy of the spleen, thymus and both white and brown adipose tissues and dilatation of the lumen due to an increase in the watery contents of the small intestine were observed as common findings in dead and moribund sacrificed animals. On histopathological examination, atrophy and fusion of the villi and dilatation of the crypts in the small intestine (Photo 1) were characteristic findings. Single cell necrosis in the spleen, thymus, lymph node, small and large intestine, liver, testes, prostate, preputial glands and Harderian glands and atrophy of hepatocytes and both white and brown adipocytes were also observed.

With doses of 1,000 and $10,000 \mu \mathrm{g} / \mathrm{kg}$, mice showed hypoactivity and a prone position. Relative polycytemia with low serum albumin concentrations, hemolysis and elevation of LDH activity and creatinine value were demonstrated. Hydrothorax and ascites in the $1,000 \mu \mathrm{g} / \mathrm{kg}$ group and red coloration and relaxation in the small intestine and lobulation in the liver in the $10,000 \mu \mathrm{g} / \mathrm{kg}$ group were macroscopically characteristic findings. Moreover, systemic enlargement and/ or red coloration of lymph nodes were observed in 1 mouse each of the two groups. Histopathological examination demonstrated hemolysis, decreased erythropoiesis (Photo 2), single cell necrosis in the thymus, spleen, lymph node, bone marrow, cardiac endothelium, liver, kidneys, prostate and seminal vesicles, and edema in the pancreas and white adipose tissue. In addition, hepatitis (Photo 3) and dilatation of glands in the glandular stomach in $10,000 \mu \mathrm{g} / \mathrm{kg}$ group and pulmonary edema in $1,000 \mu \mathrm{g} / \mathrm{kg}$ group and tubular dilatation in the kidneys in both groups were noted. The degree of injuries in the lymphoid tissues was mild in the $10,000 \mu \mathrm{g} / \mathrm{kg}$ group but strong in the $1,000 \mu \mathrm{g} / \mathrm{kg}$ group.

\section{Experiment 2}

Mice orally administered at $10,000 \mu \mathrm{g} / \mathrm{kg}$ of pierisin-1 did not show any abnormalities. Clinical laboratory tests and pathological examination also exhibited no significant effect.

\section{Experiment 3}

Time-courses of mean body weight and food consumption changes were similar to those in Experiment 1. The first clinical sign was hypoactivity, then mice adopted a hunchback position with abdominal distension, and gradually became emaciated, as seen in the $10 \mu \mathrm{g} / \mathrm{kg}$ group in Experiment 1. Relative polycythemia with a low serum albumin concentration was a characteristic hematological finding. At necropsy, atrophy of the thymus, spleen and both white and brown adipose tissue was observed. Histopathologically, basophilic change, vacuolar degeneration and single cell necrosis in the tubular epithelium, cell debris within the tubules and tubular dilatation in the kidneys (Photos $4 \mathrm{a}$ and $\mathrm{b}$ ) were characteristic findings in this experiment. The degree of these renal lesions was stronger than that of mice that received the same dose in Experiment 1. The presence of single cell necrosis in the tubular epithelium and cell debris in the uriniferous tubules indicated strong cell injury. In the small intestine, atrophy of the villi and dilatation of the crypts were observed. However, the degree of these intestinal lesions was milder than in Experiment 1. Single cell necrosis in the thymus, spleen, liver, large intestine and prostate, and atrophy of hepatocytes and both white and brown adipocytes were also observed. Single cell necroses of hepatocytes were particularly prominent in mice showing higher activity of both AST and ALT.

\section{Experiment 4}

Almost all abnormalities are limited to rats of the 
Acute toxicity of pierisin-1.

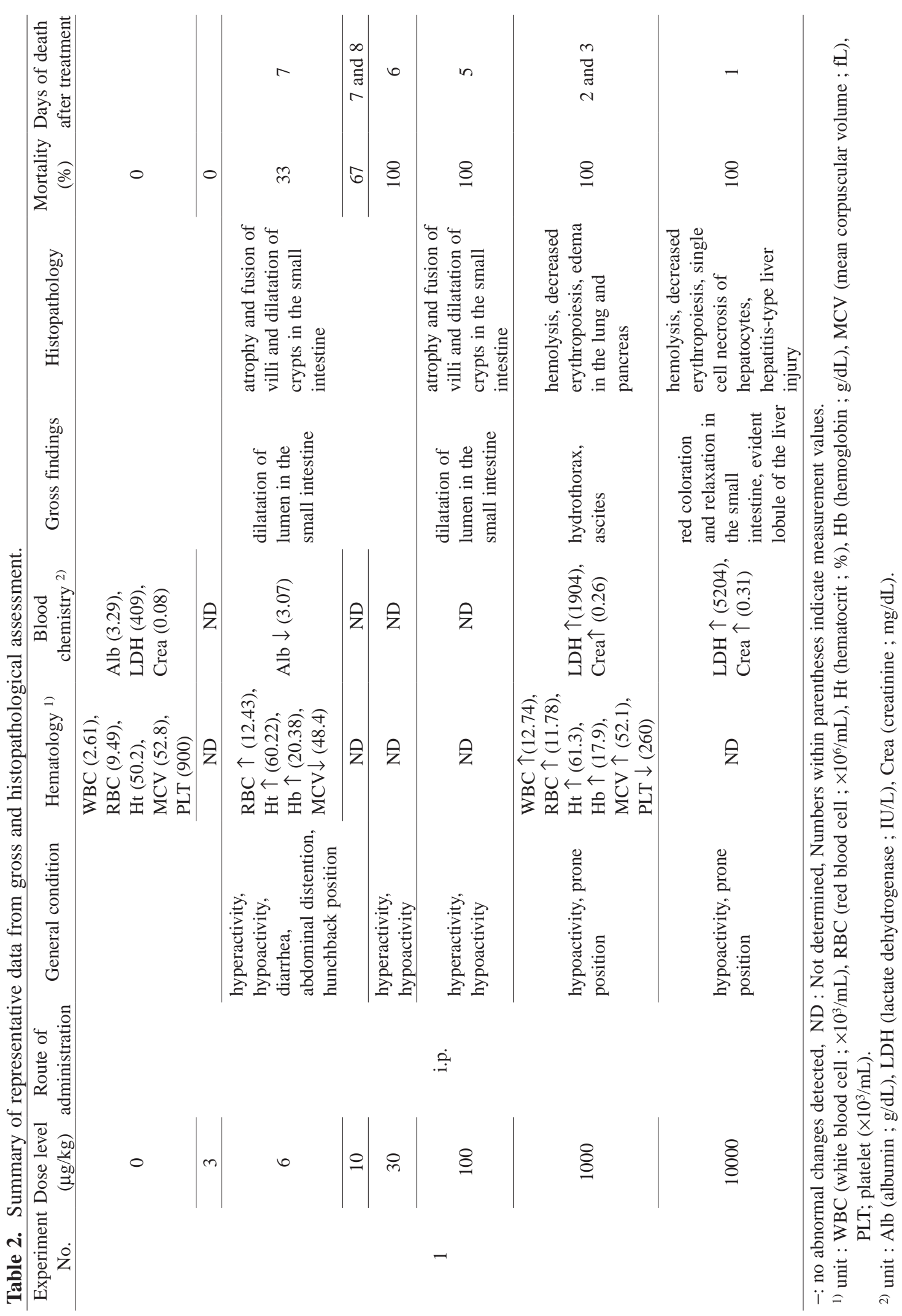


A. SHIGA et al.

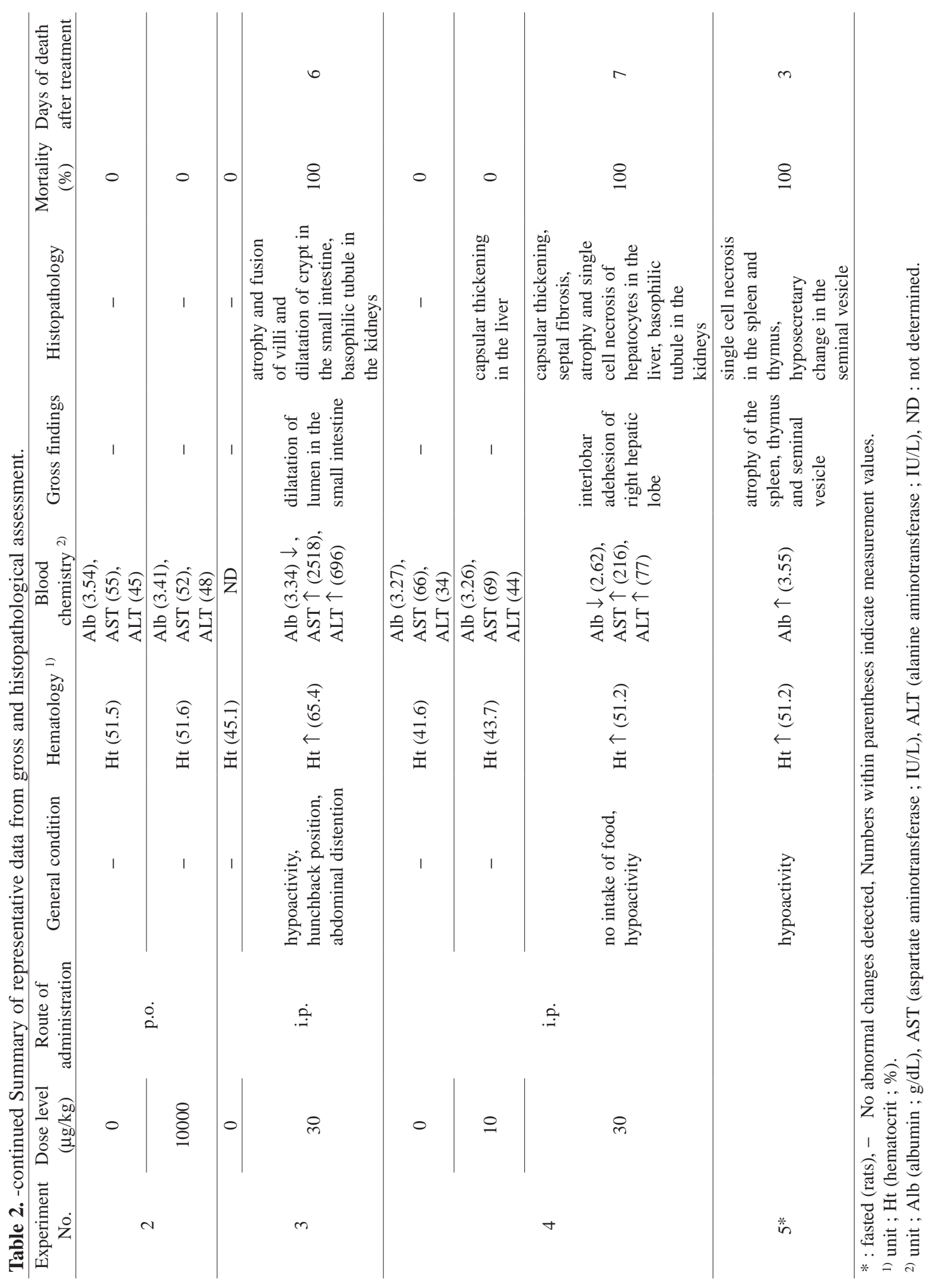


Acute toxicity of pierisin-1.

$30 \mu \mathrm{g} / \mathrm{kg}$ group. The body weights gradually decreased and the rate of suppression of body weight gain was about $28 \%$, and there was no food intake seen as the first clinical sign for 4 days after dosing. Thereafter, rats showed hypoactivity, lacrimation and piloerection. Relative polycythemia with low serum albumin concentration and elevation of AST and ALT activities were found by clinical laboratory tests. At necropsy,

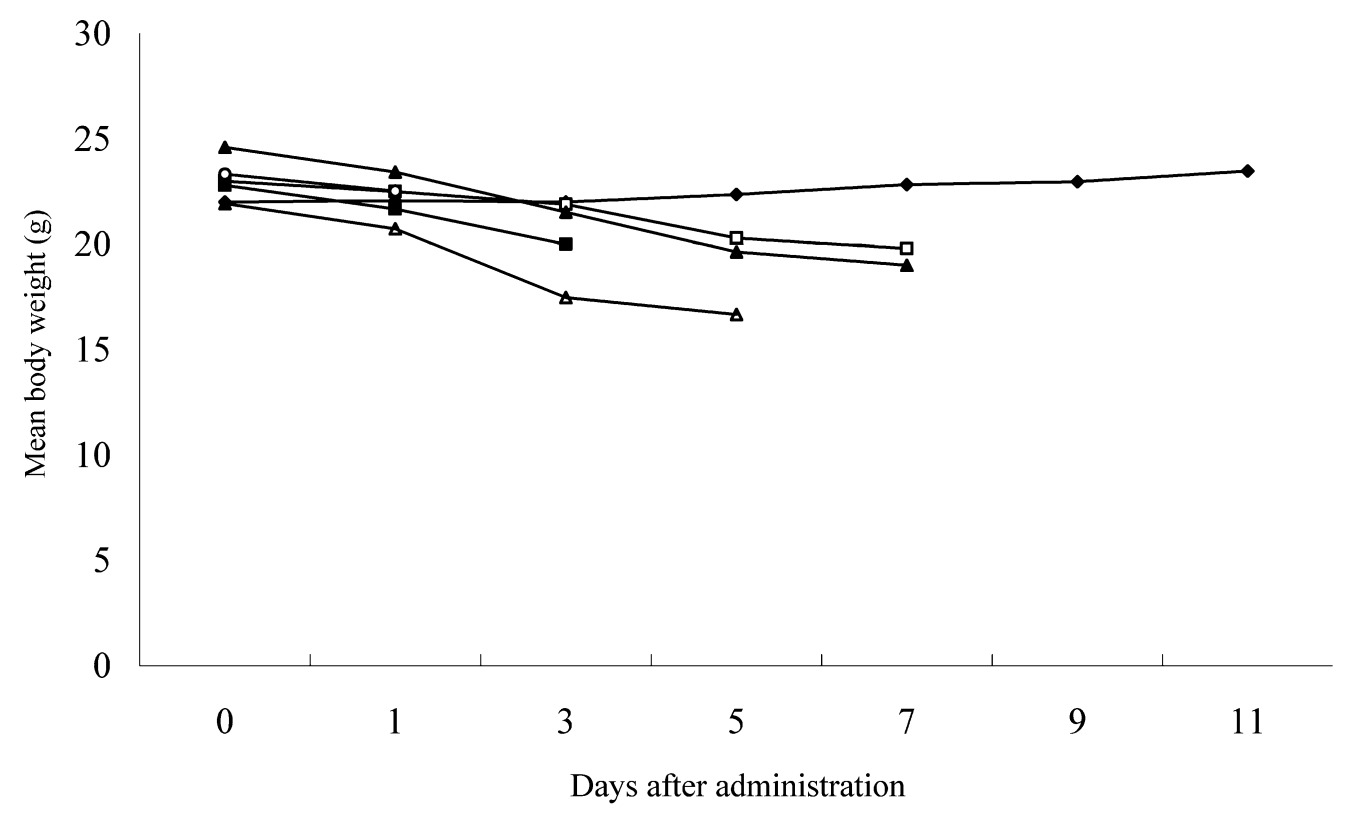

Fig. 1. Time-course of change in mean body weights (Experiment 1).

$\mathrm{X}$ axis : Days after administration, $\mathrm{Y}$ axis : Mean body weight $(\mathrm{g})$,

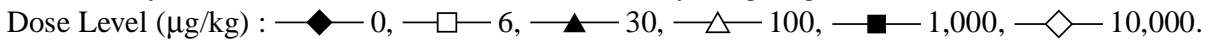

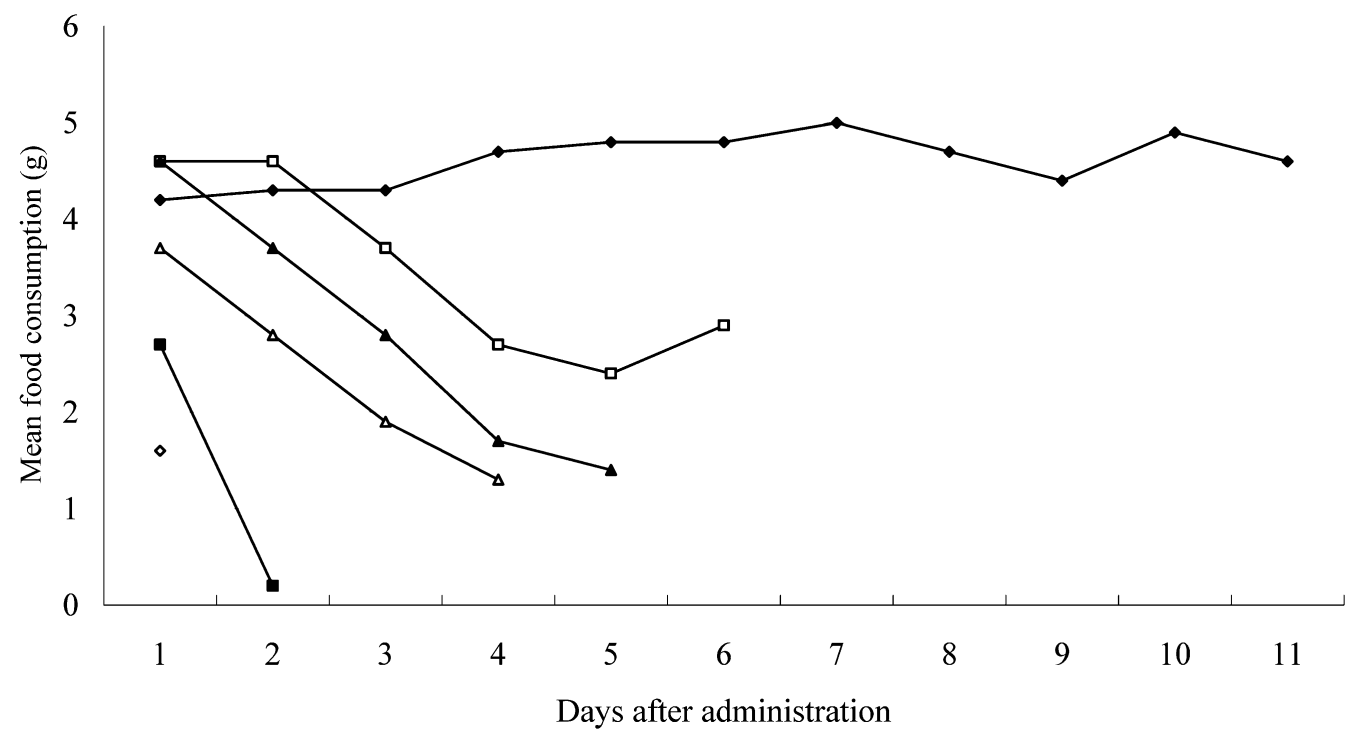

Fig. 2. Time-course of change in mean food consumption (Experiment 1).

$\mathrm{X}$ axis : Days after administration, $\mathrm{Y}$ axis : Mean food consumption $(\mathrm{g})$,

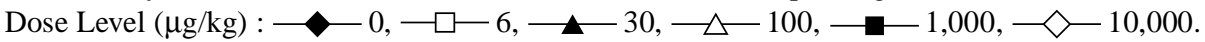


A. SHIGA et al.

interlobar adhesion in the right lobe of the liver was characteristic (Photo 5). The other macroscopic finding was atrophy of the thymus, spleen, seminal vesicle and both white and brown adipose tissue. Histopatho- logical examination demonstrated septal fibrosis, capsular thickening and single cell necrosis of subcapsular hepatocytes in the liver (Photo 6) and basophilic tubules in the kidney. The sudden stop in food intake

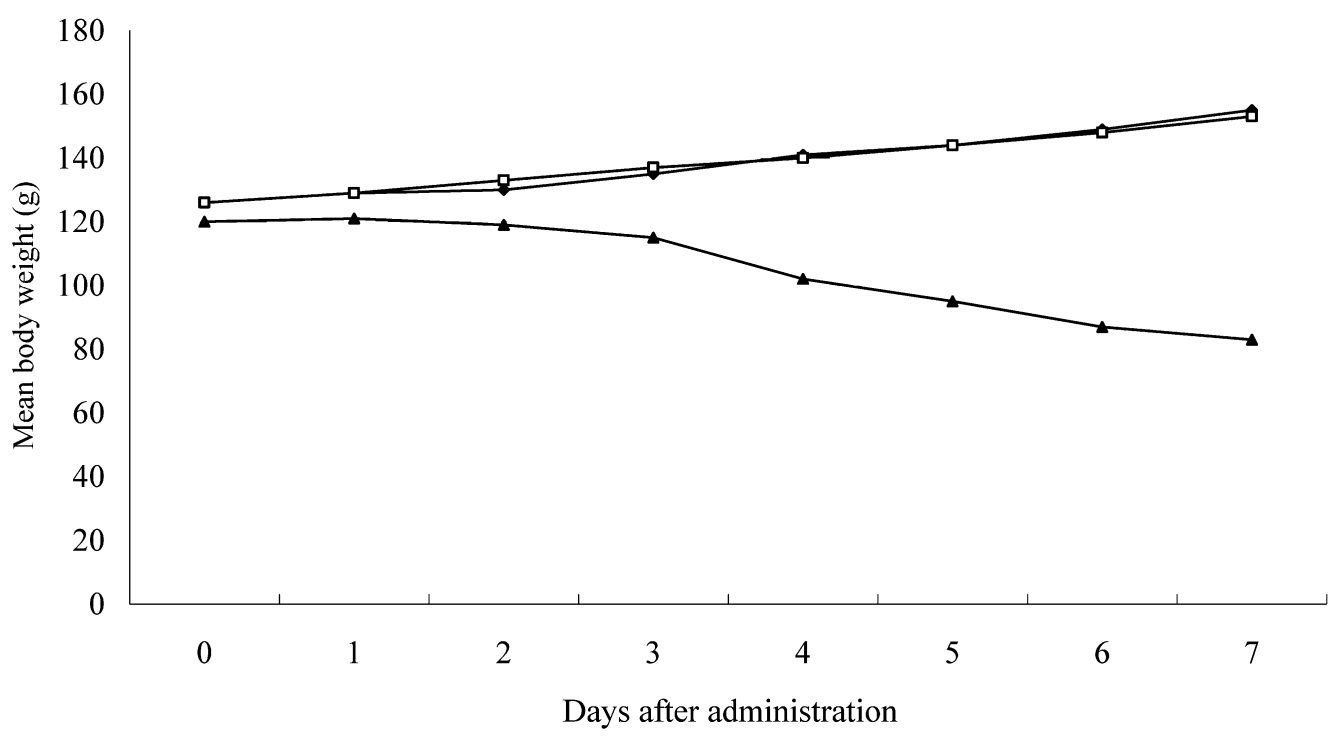

Fig. 3. Time-course of change in mean body weights (Experiment 3) $\mathrm{X}$ axis : Days after administration, Y axis : Mean body weight (g), Dose Level $(\mu \mathrm{g} / \mathrm{kg})$ : $\square-10, \longrightarrow 30$.

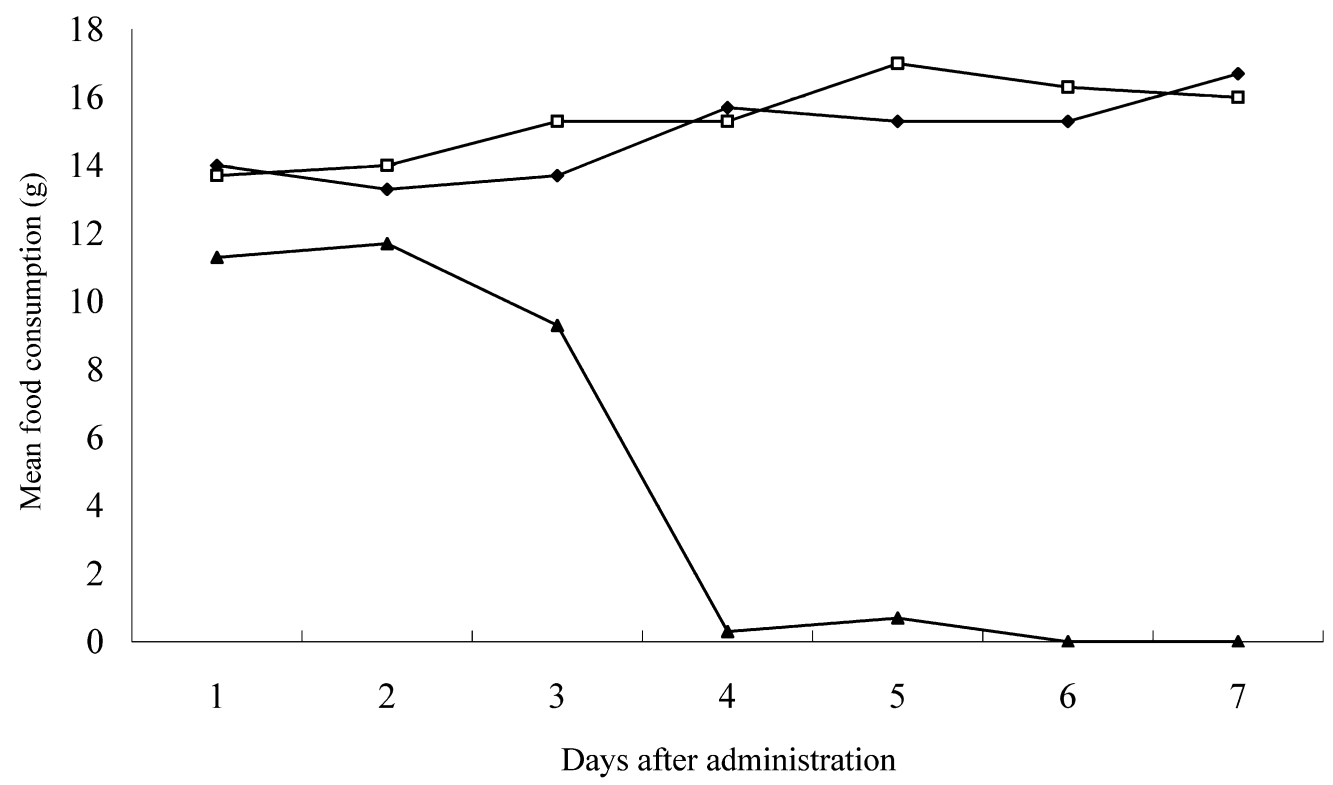

Fig. 4. Time-course of change in mean food consumption (Experiment 3) $\mathrm{X}$ axis : Days after administration, $\mathrm{Y}$ axis : Mean food consumption $(\mathrm{g})$, Dose Level $(\mu \mathrm{g} / \mathrm{kg})$ : $\longrightarrow 0, \square \longrightarrow \mathbf{\Delta} \quad 30$. 
Acute toxicity of pierisin-1.

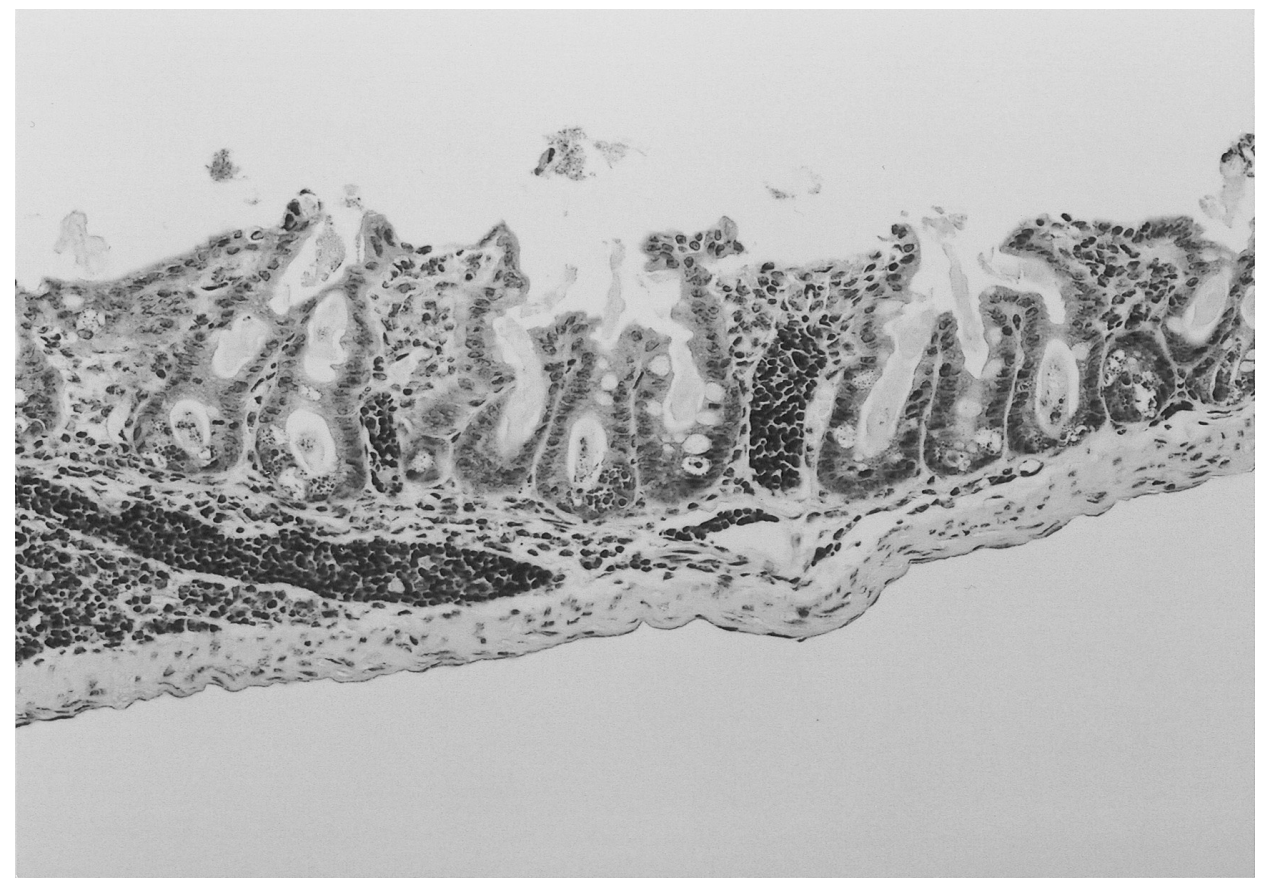

Photo 1. BALB/c mouse, $10 \mu \mathrm{g} / \mathrm{kg}$ group (Experiment 1),

Ileum ; Note the atrophy and fusion of villi and dilatation of the crypts, HE stain, $\times 200$.

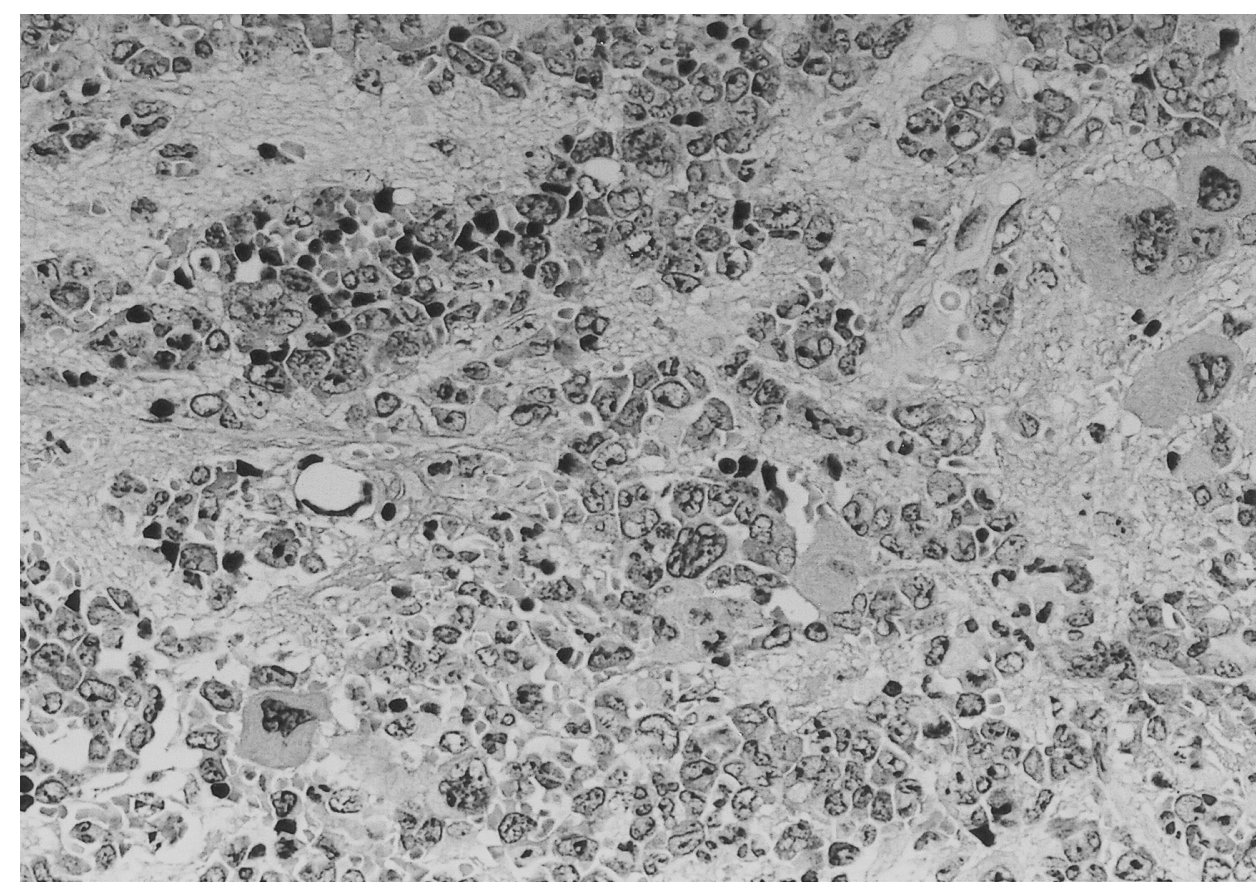

Photo 2. BALB/c mouse, $1000 \mu \mathrm{g} / \mathrm{kg}$ group (Experiment 1),

Bone marrow (sternum); Note hemolysis and decreased erythropoiesis, HE stain, $\times 350$. 
suggested an effect on the center regulating food intake, but there were no histological abnormalities detected in the ventromedial nucleus of hypothalamus; a regulatory center of food intake. As other findings, hemorrhage and decreased hematopoiesis in the bone marrow, atrophy of the thymus and spleen and hyposecretory change in the seminal vesicle were observed. Capsular thickening in the liver was also observed in both males and females in the $10 \mu \mathrm{g} / \mathrm{kg}$ group.

\section{Experiment 5}

Fasted mice showed atrophy of the spleen, thymus and both white and brown adipose tissue, and dilatation of the lumen in the gallbladder on gross examination. Histopathological examination demonstrated single cell necroses in the spleen, lymph node, thymus, liver, prostate and preputial glands, atrophy of hepatocytes, ulceration in the glandular stomach, and atrophy of hepatocytes and both white and brown adipocytes.

In fasted rats, relative polycythemia with high serum albumin concentration, hemorrhage and decreased hematopoiesis in the bone marrow, single cell necrosis in the thymus, atrophy of the spleen and both white and brown adipocytes and hyposecretory changes in the seminal vesicles were observed.

\section{DISCUSSION}

Pierisin-1 toxicity in mice in the present study increased in degree and incidence of lesions in a doserelated manner. A variety of toxic effects including a decrease in food intake, malabsorption due to injury of the intestinal mucosa at doses of $6-100 \mu \mathrm{g} / \mathrm{kg}$, and hepatic and renal injury, hemolysis and circulatory disturbance at doses of 1,000 and $10,000 \mu \mathrm{g} / \mathrm{kg}$ were considered to be due to direct toxicity of pierisin-1 in $\mathrm{BALB} / \mathrm{c}$ mice and the cause of death varied depending on the dose level. Elevated LDH was considered to be due to hemolysis, this finding being noted in mice without abnormalities in the heart, liver or brain. Expression of stronger renal toxicity to ICR mice than $\mathrm{BALB} / \mathrm{c}$ mice suggests that there are strain differences in the toxicity of pierisin-1 in mice. The cause of death in F344 rats could be considered to be emaciation due to no intake of food, although toxic effects were observed in the liver and kidney.

Recently, globotriosylceramide (Gb3) and globotetraosylceramide (Gb4), which are receptors for verotoxin from Shigera bacteria, were proven to serve

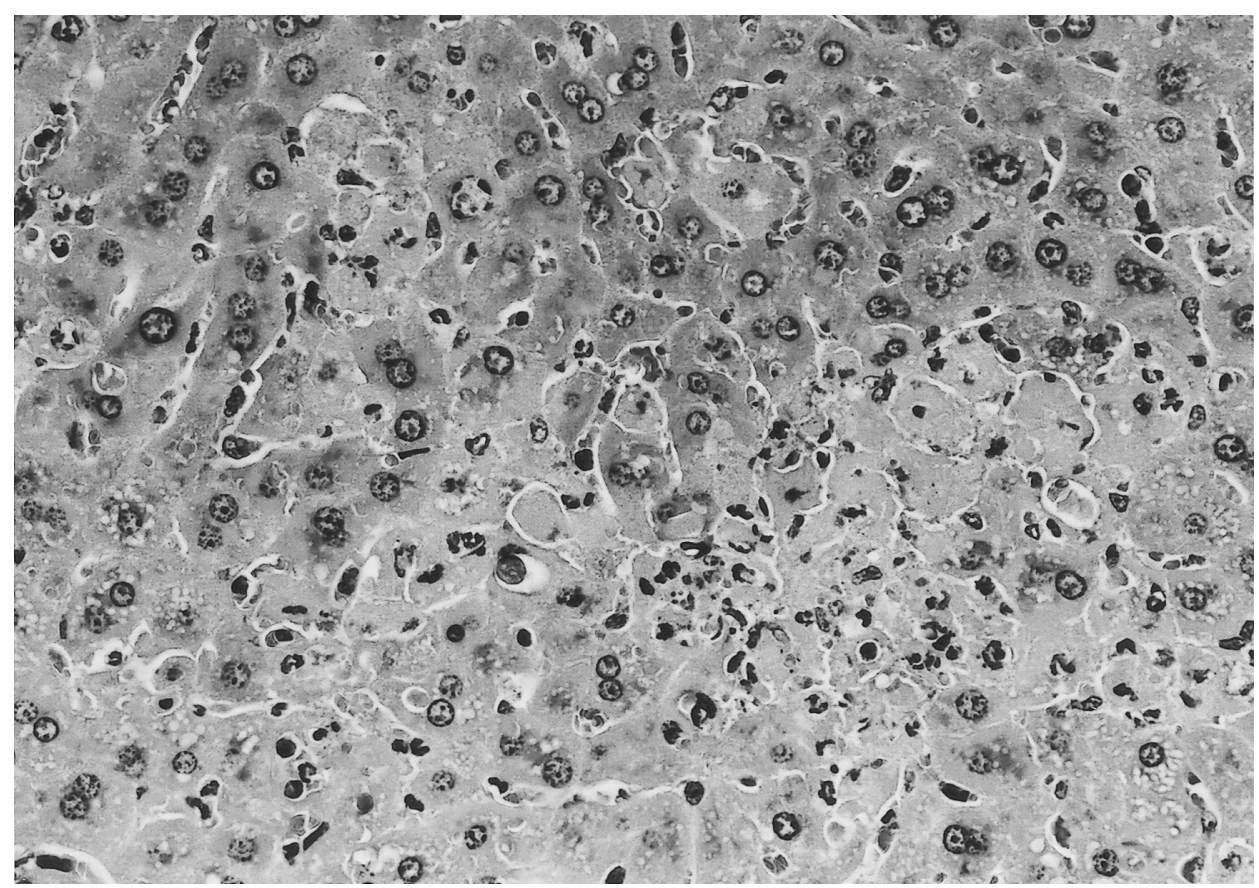

Photo 3. BALB/c mouse, $10000 \mu \mathrm{g} / \mathrm{kg}$ group (Experiment 1), Liver ; Hepatitis-type hepatic injury, HE stain, $\times 400$. 
Acute toxicity of pierisin-1.

as pierisin-1 receptors (Matsushima-Hibiya et al., 2003). Gb3 is distributed in the vascular endothelium, spleen, kidney, mucosa of the small intestine, while Gb4 is found in the kidney, spleen, erythrocytes. In fact, characteristic findings in the present experiments were observed in the small intestine, kidney and erythrocytes and in vitro studies have demonstrated that the amount of receptors in cells is one of the main factors affecting sensitivity of cells (Matsushima-Hibiya et al., 2003).

Gradual decrease in body weight gain is also induced by 2,3,7,8-tetrachlorodibenzo- $p$-dioxin (TCDD) and the sign is referred to the wasting syndrome, which is related to altered retinoid homeostasis (Fletcher et al., 2001). The heterodimeric partner of peroxisomal proliferator activated receptor $\gamma(\operatorname{PPAR} \gamma)$ receptor is the retinoid $X$ receptor ( $R X R$ )
(Mangelsdorf and Evans, 1995). PPAR $\gamma$ agonist induces atrophy of adipose tissue and its side effect is systemic fluid retention (Guan et al., 2005), which are seen in the present study. However, the roles of retinoid and/or PPAR $\gamma$ in pierisin-treated animals were unknown.

Lesions which cannot be explained by the presence of receptors induce relative polycythemia with a low albumin concentration in mice and rats, hydrothorax, ascites, hepatitis-like injuries in mice at doses of 1,000 or $10,000 \mu \mathrm{g} / \mathrm{kg}$ and the sudden stop of food intake in rats. Pierisin-1 is a toxin derived from insects and that is anticipated as a new candidate for cancer therapy. Likewise, immunotoxins which are chimeric molecules consisting of cell binding ligands and toxins or their subunits are used for chemotherapy. However, they are known to induce vascular leak syndrome
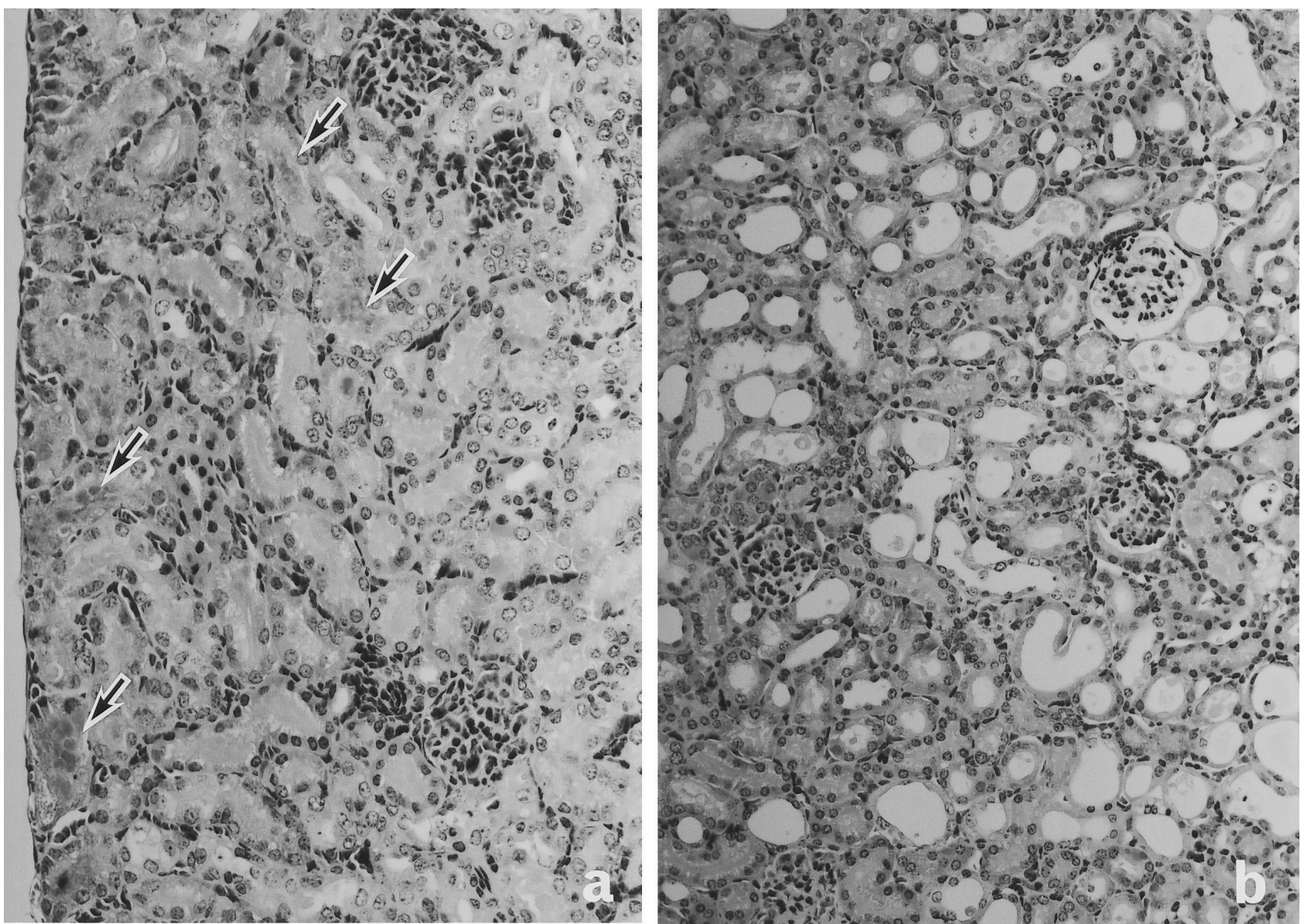

Photo 4. ICR mouse, $30 \mu \mathrm{g} / \mathrm{kg}$ group (Experiment 2),

Kidney ; (a) Basophilic change of the tubular epithelium and cell debris in the tubules(arrows), HE stain, $\times 192$.

(b) Dilatation of uriniferous tubules, HE stain, $\times 288$. 


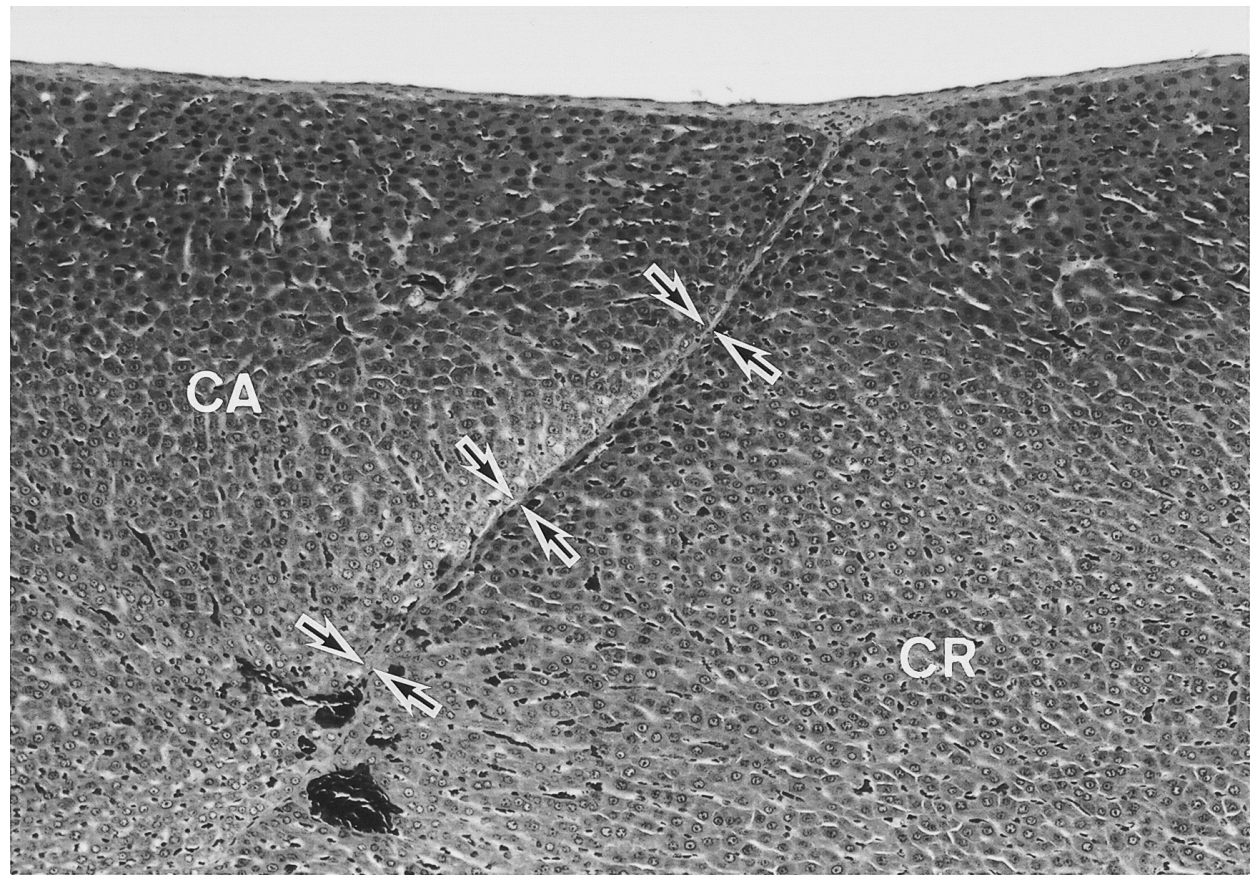

Photo 5. F344 rat, $30 \mu \mathrm{g} / \mathrm{kg}$ group (Experiment 3),

Liver ; Fibrous adhesion (arrows) between the cranial (CR) and caudal part (CA) of the right lobe, HE stain, $\times 300$.

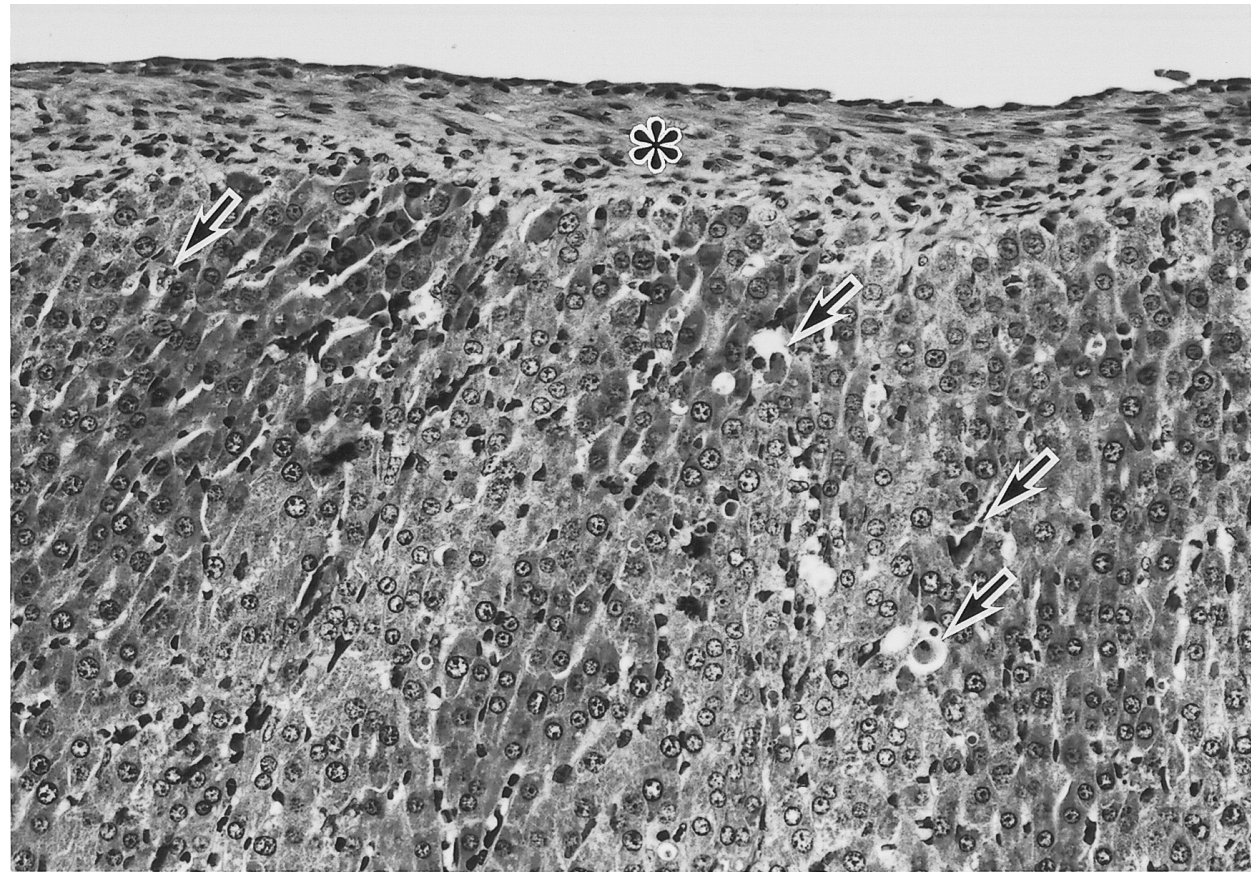

Photo 6. F344 rat, $30 \mu \mathrm{g} / \mathrm{kg}$ group (Experiment 3),

Liver ; Capsular thickening $\left(^{*}\right)$ and single cell necrosis of subcapsular hepatocytes (arrows), HE stain, $\times 200$. 
Acute toxicity of pierisin-1.

(VLS), characterized with hypoalbuminemia, edema and body weight gain, although their mechanism is yet unclear (Vietta et al., 1993). In the present study, these human VLS-like symptoms were also observed in mice treated with $1,000 \mu \mathrm{g} / \mathrm{kg}$ of pierisin-1. Moreover, relative polycythemia was characteristic of hematological findings, although serum albumin concentration was low. This phenomenon may be suggested to be an increase in vascular permeability as seen in VLS, rather than a mere dehydration.

Immunotoxins, BR96 sFv-PE40 can induce VLS in rats as established as an animal model of VLS (Siegall et al., 1994). BR96 sFv-PE40-induced VLS are known to be suppressed by pretreatment of dexamethazone (Siegall et al., 1994) and nonsteroidal antiinflammatory drugs (NASIDs) (Siegall et al., 1997) and but not by histamine or serotonine (Siegall et al., 1994, 1997) or not reproduced in athymic rats (Siegall et al., 1994), suggesting a lack of T-cell response. Interleukin-2 (IL-2) induces VLS in mice and tumor necrosis factor- $\alpha$ (TNF- $\alpha)$ plays a central role in their mechanism (Rosenstain et al., 1986; Dubinett et al., 1994). Immunosuppression of mice by pretreatment with irradiation (500 rad) or by injection of cyclophosphamide or by concurrent use of cortisone acetate reduced or eliminated markedly the development of VLS (Onda et al., 2000). Similarly, VLS was not observed in nude mice receiving IL-2 (Onda et al., 2000). Based on these findings, immune response was considered to have an important role in the pathogenesis of VLS. In fact, lymphoid organs injuries were evident in mice showing VLS-like symptoms in the present study.

Hepatic injuries are also one of the side effects due to immunotoxins (Vietta et al., 1993). As for immunotoxins-induced hepatic injuries, TNF- $\alpha$ produced by Kupffer cells has an important role in pathogenesis (Schilling et al., 1992). TNF- $\alpha$ and agonistic Fas are known to have also anticancer activity and administration of high dose TNF- $\alpha$ in humans (Schilling et al., 1992) and anti-Fas antibody in mouse (Ogasawara et al., 1993) is reported to cause hepatotoxicity by an unknown mechanism. In mice, an administration of BR96 sFv-PE40 induced no VLS but hepatic injuries (Siegall et al., 1994). In the present study, large administration of pierisin caused only hepatitis-type hepatic injury in mice without showing lymphoid organs injuries. This change was considered a dose-limited toxicity and host immune response may be participated in their pathogenesis.

Mice administered orally $10,000 \mu \mathrm{g} / \mathrm{kg}$ of pieri- sin-1 did not exhibit any abnormal changes in any of the parameters examined, even though the dose is 2,000 times the $\mathrm{LD}_{50}$ by the i.p. route. Thus the cytotoxic activity of pierisin-1 may be lost with proteolytic enzyme digestion. Similar toxicity appeared with administration via i.v. route. Approximately 50-fold dose were requires for exertion of pierisin-1 toxicity by s.c. routes. Therefore, direct entrance of pierisin- 1 into the blood may be needed to exert toxicity.

In pierisin-1-treated rats, the sudden stop in food intake, relative polycythemia with low serum albumin concentration and interlobar adhesion of the right lobe in the liver were characteristic findings lacking in fasted rats. However, the cause for the loss of appetite could not be identified morphologically, although immune cytokines such as interleukin-1 or interferon$\alpha$ are suspected to play a role in anorexia (Hori et al., 1991). The pathogenesis of the interlobar adhesion of hepatic right lobe was intriguing given the lack of hepatic necrosis. It pointed to a remodeling of the hepatic architecture.

In conclusion, pierisin- 1 shows species and strain differences in toxicity to animals. Investigation of possible causative factors such as receptor for pierisin-1 would be very helpful to understand the toxic mechanism of pierisin- 1 in animals.

\section{ACKNOWLEDGMENT}

The authors are grateful to Drs. Takashi Kanazawa, Masahiko Watanabe (Faculty of pharmacy, Shujitsu University) and Keiji Wakabayashi, National Cancer Center Research Institute, for supplying Pierisin-1 and Mrs M. Tsuchiya, An-Pyo Center, for assistance in preparation of this paper.

\section{REFERENCES}

Dubinett, S.M., Huang, M., Lichitenstein, A., McBride, W.H., Wang, J., Markovitz, G., Kelley, D., Grody, W.W., Mintz, L.E. and Dhanani, S. (1994): Tumor necrosis factor- $\alpha$ plays a central role in interleukin-2-induced pulmonary vascular leak and lymphocyte accumulation. Cell Immunol., 157, 170-180.

Fletcher, N., Hanberg, A. and Hakansson, H. (2001): Hepatic vitamin A depletion is a sensitive marker of 2,3,7,8-tetrachlorodibenzo- $p$-dioxin (TCDD) exposure in four rodent species. Toxi. Sci., 62, 166-175.

Guan, Y.-F., Hao, C., Cha, D.R., Rao, R., Lu, W., 
Koran, D.E., Magnuson, M.A., Redha, R., Zhang, Y. and Breyer, M.D. (2005): Thiazolidinediones expand body fluid volume through PPAR $\gamma$ stimulation of ENaC-mediated renal salt absorption. Nature Med., 11, 861-866.

Hori, T., Nakashima, T., Take, S., Kaizuka, Y., Mori, T. and Katafuchi, T. (1991): Immune cytokines and regulation of body temperature, food intake and cellular immunity, Brain Res. Bull., 27, 309313.

Kanazawa, T., Watanabe, M., Matsushima-Hibiya, Y., Kono, T., Tanaka, N., Koyama, K., Sugimura, T. and Wakabayashi, K. (2001): Distinct roles for the $\mathrm{N}$ - and $\mathrm{C}$ - terminal regions in the cytotoxicity of pierisin-1, a putative ADP-ribosylating toxin from cabbage butterfly, against mammalian cells. Proc. Natl. Acad. Sci. U.S.A., 98, 2226-2231.

Kanazawa, T., Kono, T., Watanabe, M., MatsushimaHibiya, Y., Nakano, T., Koyama, K., Tanaka, N., Sugimura, T. and Wakabayashi, K. (2002a): Bcl-2 blocks apoptosis caused by pierisin-1, a guanine-specific ADP-ribosylating toxin from the cabbage butterfly. Biochem. Biophys. Res. Commun., 296, 20-25.

Kanazawa, T., Watanabe, M., Kanzawa, F. Matsushima-Hibiya, Y., Koyama, K., Tanaka, N., Sugimura, T. and Wakabayashi, K. (2002b): Pierisin-1 from cabbage butterfly suppresses HeLa tumor growth in nude mice. Proc. Jpn. Acad., 78B, 305-308.

Kono, T., Watanabe, M., Koyama, K., Kishimoto, T., Fukushima, S., Sugimura, T. and Wakabayashi, K. (1999): Cytotoxic activity of pierisin-1, from the cabbage butterfly, Pieris rapae, in various human cancer cell lines. Cancer Lett., 137, 7581.

Koyama, K., Wakabayashi, K., Matsutani, M., Koiwai, K., Watanabe, M., Yamazaki, S., Kono, T., Miki, K. and Sugimura, T. (1996): Presence in Pieris rapae of cytotoxic activity against human carcinoma cells. Jpn. J. Cancer Res., 87, 1259-1262.

Mangelsdorf, D.J. and Evans, R.M. (1995) : The RXR heterodimers and orphan receptors. Cell, 83, 841-850.

Matsushima-Hibiya, Y., Watanabe, M., Hidari, K.I., Miyamoto, D., Suzuki, Y., Kasama, T., Koyama, K., Sugimura, T. and Wakabayashi, K. (2003): Identification of glycosphingolipid receptors for pierisin-1, a guanine-specific ADP-ribosylating toxin from the cabbage butterfly. J. Biol. Chem.,
278, 9972-9978.

Ogasawara, J., Watanabe-Fukunaga, R., Adachi, M., Matsuzawa, A., Kasugai, T., Kitanuma, Y., Itoh, N., Suda, T. and Nagata, S. (1993): Lethal effects of the anti-Fas antibody in mice. Nature, 364, 806-809.

Onda, M., Willingham, M., Qang, Q.-C., Kreitman, R.J., Tsutsumi, Y., Nagata, S. and Pastan, I. (2000): Inhibition of TNF- $\alpha$ produced by Kupffer cells protects against the nonspecific liver toxicity of immunotoxin anti-Tac(Fv)PE38, LMB-2. J. Immunol., 165, 7150-7156.

Rosenstein, M., Ettinghausen, S.E. and Rosenberg, S.A. (1986): Extravasation of intravascular fluid mediated by the systemic administration of recombinant interleukin-2. J. Immunol., 137, 1735-1742.

Schilling, P.J., Murray, J.L. and Markowitz, A.B. (1992): Novel tumor necrosis factor toxic effects. Pulmonary hemorrhage and severe hepatic dysfunction. Cancer, 69, 256-260.

Siegall, C.B., Liggitt, D., Chase, D., Mixan, B., Sugai, J., Davidson, T. and Steinitz, M. (1997): Characterization of vascular leak syndrome induced by the toxin component of Pseudomonas exotoxinbased immunotoxins and its potential inhibition with nonsteroidal anti-inflammatory drugs. Clin. Cancer Res., 3, 339-345.

Siegall, C.B., Liggitt, D., Chase, D., Tepper, M.A. and Fell, H.P. (1994): Prevention of immunotoxinmediated vascular leak syndrome in rats with retention of anticancer activity. Proc. Natl. Acad. Sci. U.S.A., 91, 9514-9518.

Takamura-Enya, T., Watanabe, M., Totsuka, Y., Kanazawa, T., Matsushima-Hibiya, Y., Koyama, K., Sugimura, T. and Wakabayashi, K. (2001): Mono(ADP-ribosyl)ation of 2'-deoxyguanosine residue in DNA by an apoptosis-inducing protein, pierisin-1, from cabbage buttefly. Proc. Natl. Acad. Sci. U.S.A., 98, 12414-12419.

Totsuka, Y., Kawanishi, M., Nishigaki, R., Matsukawa, T., Yagi, T., Takamura-Enya, T., Watanabe, M., Sugimura, T. and Wakabayashi, K. (2003): Mutation analysis of HPRT and supF genes caused by pierisin-1, a guanine-specific ADP-ribosylating toxin derived from the cabbage butterfly. Chem. Res. Toxicol., 16, 945952.

Vietta, E.S., Thorpe, P.E. and Uhr, J.W. (1993): Immunotoxins: Magic bullets or misguided missiles ? Trends Pharmacol. Sci., 14, 148-154. 
Acute toxicity of pierisin-1.

Watanabe, M., Kono, T., Koyama, K., Sugimura, T. and Wakabayashi, K. (1998): Purification of pierisin-1, an inducer of apoptosis in human gastric carcinoma cells, from cabbage butterfly, Pieris rapae. Jpn. J. Cancer Res., 89, 556-561.

Watanabe, M., Kono, T., Matsushima-Hibiya, Y., Kanazawa, T., Nishikawa, N., Kishimoto, T.,
Koyama, K., Sugimura, T. and Wakabayashi, K. (1999): Molecular cloning of an apoptosisinducing protein, pierisin-1, from cabbage butterfly : Possible involvement of ADP-ribosylation in its activity. Proc. Natl. Acad. Sci. U.S.A., 96, 10608-10613. 